\title{
Explorationson Seizing Opportunities in the Tide of Silver Economy via Intelligent Old-Age Care System from International Perspective
}

\author{
Yanhong Wu*, Yahao Xu, Jingxing Wang \\ Foreign language school, Zhejiang Ocean University, china
}

*Corresponding Author: Yanhong Wu, Foreign language school, Zhejiang Ocean University, china

\begin{abstract}
In the $21^{\text {st }}$ century, there is an intertwined background between the world population aging and the rapid development of various information technologies, which includes the world Internet of Things, cloud computing, big data, etc. To propel the development of the intelligent old-age care system is a vital way to positively face and handle the aging of the population and to accelerate the development of old-age programs and industries. Being positioned at the 'tip' of the state innovation system, some islands, though tagged as important and special geographic entity of the ocean, haven't been attached much attention, resulting an incomplete embodiment of their worth, which includes their enormous rights, resources, ecology, economy and so on. Studying the case of Zhoushan, this thesis will analyze the strengths and weaknesses of creating an international superb place for intelligent old-age care by considering the advantages of being an international island tourism area against the background of the world population aging and the big data era. The purpose of this thesis is to build a new identity for Zhoushan, grasp the opportunity in the tide of silver economy and offer advice and suggestions for stimulating the new development of economy.
\end{abstract}

Keywords: Aging, Big data, Intelligent Old-age Care System.

\section{INTRODUCTION}

By the definition of the United Nations, a country or region enters into an "aging society" when the proportion of its population that is over 65years old exceeds $7 \%$ of the total, while when this proportion reaches $14 \%$, it signifies the advent of "aged society", and $20 \%$ marks a hyper-aged society.

Pointed by the World Population outlook 2019 released by the United Nations recently, the population structure will continue to age as a whole. In 2019, the proportion of the elderly aged above 65 accounts for $1 / 6$ of the global population, which will rise to $1 / 11$ by 2050 . At present, China's aging rate is $12.0 \%$, ranking 57 th in the world; it is expected to be $20.7 \%$ in 2035, 44th in the world, $26.1 \%$ in 2050, and 33rd in the world. Statistics shows that the trend of population aging in Zhoushan City is developing rapidly. The total number of the elderly over 60 years old increased from 198,600 at the end of 2012 to 256,900 at the end of 2017, and the aging coefficient increased from $20.36 \%$ at the end of 2012 to $26.44 \%$ at the end of 2017 , presenting a tendency of increasing year by year. The aging level of Zhoushan has exceeded greatly the national average level. Since we entered the aging society, our elderly population has presented some more evident traits and trends, such as the large population base with a fast velocity of increase and the inclination of senility, disability and empty nest, aggravating the problem of old-age care with an additional reason, which is that our family has become smaller, while one of our national conditions is that we are aging before we are getting rich.

Thus, in the context of world population aging, our endeavor to transform the aging population into a favorable factor for sustaining socio-economic development will make a positive difference in the profound process of population aging's integration with series of national strategies and major moves, such as the innovation-driven development, rural revitalization and sustainable development, and will become a new direction to actively ponder and cope with population aging while keep on developing

The notion of Smart City was introduced by IBM after it put forward the idea of Smart Earth in State China Relations Council held in 2008, New York. For now, there has not been so much understanding 
towards the terms like "smart" or "intelligent" in our country. In Solutions to build new aging communities digitally and homely issued by Hu LiMing in 2007, the concept of digitalized old-age care was introduced, followed by the idea of information alized old-age care, technological old-age care and networked elderly health care. Through a series of conceptual changes, the intelligent old-age care has finally received recognition of various circles and becomes a new idea to solve the aging issue.

Based on the assorted information technologies like the Internet of Things, Mobile Internet, Big data and Cloud Computing, the intelligent old-age care system is an advanced supporting model for the aged. Being different from the old traditional model, the intelligent old-age care system has more informational zed services, management modes and more modernized business models to provide the aged with more healthy, convenient, secure and efficient services for their lives. As the first nationallevel new district of islands and a crucial component of the Yangtze River Delta urban agglomerations, the Zhoushan archipelago, with the tide of silver economy approaching, is competently capable of presenting its strength at the new economic development point, promoting the new economic development of the new district and improving people's satisfaction and well-being with life.

\section{ANALYSIS}

\subsection{Abundant Natural and Human Resources}

As the largest archipelago in China, Zhoushan is located in the East China Sea at the south of the Yangtze River Estuary and the outer edge of Hangzhou Bay. Surrounded by the sea, it has a subtropical monsoon climate and is warm in winter and cool in summer. The environment there is mild and humid, and has sufficient light. Moreover, its air quality frequently ranks among the top ten cities in China, with the negative oxygen ion, a substance the medical field gives "vitamin oxygen", "longevity element", "air vitamin" and all that laudatory titles to, richly found in the air. The representative color of blue in Zhoushan's sky has already symbolized the city itself.

Known as Haizhongzhou, the islet amid the sea, since the ancient time, Zhoushan has a long history and many special landscapes as a sea island, which gives it infinite fascination and makes it particularly enchanting. The blue sky, aquamarine sea, green island, gold beaches and white waves there are what embody the dominant tone of its eco-tourism environment. Being praised as "the Buddhist Kingdom on the sea" and featured by sea, fishing, city, island, port, sailing and business, it combines island scenery, marine culture and Buddhist cultureas a whole. The prestigious Buddhist culture also plays a significant part in attracting the aged for meditation and mind-cultivating.

\subsection{Supportive National Policies}

In accordance with the spirit in the Opinions of the State Council on accelerating the development of the elderly care service industry ( (2013) No.35 Document of State Council ) and Opinions of the People's Government of Zhejiang Province on accelerating the development of elderly care service industry ( (2014) No.13 Document of the People's government of Zhejiang Province ), the Implementation opinions of Dinghai District People's Government of Zhoushan City on accelerating the development of elderly care service industry (2014) No.31 Document of Dinghai District People's Government gives its advice on developing intelligent old-age care system creatively. The advancement on the construction of information management system and building of information management system for old-age care has also been mentioned to commonly create an information base in the community for empty-nested families and elderly people who live alone, suffer from deformity and diseases or are in advanced age. In doing so, the dynamic management of information of the elderly people can be gradually achieved.

Action plans for enriching, benefiting, stabilizing people of Zhejiang Province (2018) No.22 Document of the people's government of Zhejiang Provinceis executing intelligent old-age care system. The standards of intelligent old-age care system should be created. The network for old-age care services should be formed by means of adopting the theory and idea of "Internet plus" and "Internet of Things plus". The information touching the old-age care services could be released regularly by making use of the health records of the aged provided by communities and by building the very database for the aged. The functions of the platform for the home old-age care services 
should be improved and perfected, and for the aged, they should be equipped more widely with the intelligent terminal devices. In doing so, the emergency services and special urgent aids can be provided. The introductions of all those policies have necessarily guaranteed the implementation of the plans for the intelligent old-age care system.

\subsection{Promising Market Prospects}

As far as the locality of Zhoushan is concerned, its population has presented negative growth by the end of 2018 and its degree of the aged phenomenon has far exceeded the national average level. For example, the registered population of TaohuaIsland in Zhoushan is 16356, of which the population over 60 years old is 5327 , accounting for $32.5 \%$ of the total. Since the proportion of the population aged over 60 of this area's total population is far more than $10 \%$, we can infer that the Taohua Island has an old population age structure and is currently at the stage of severe aging.

As far as other places are concerned, Zhoushan has relatively strong geographic advantages with Shanghai, Hangzhou, Ningbo and other large and medium-sized cities nearby. Other than that, where it is situated is adjacent to the hinterland of Yangtze River delta and is faced with the Pacific, which makes it the pivotal confluence between China's north-south coastal routes and Yangtze River waterway. As a doorway at the sea, it even becomes the avenue for the opening-upof the Yangtze River basin and delta. Moreover, the coastal regions adjoining Zhoushan enjoy a fast development in their economy and the people there have a relatively high average income. Currently, some models of old-age care relying on the innovative development are burgeoning worldwide, such as the model of traveling while serving for the aged and the model of traveling like migratory birds while serving for the aged, which brings the oncoming international intelligent old-age care center of Zhoushan a respectable volume of passenger.

\subsection{The Facilities for Intelligent Old-Age Care System are Satisfactory}

Since November, 2014, Zhoushan has made preparations for the founding of information platform for intelligent old-age carein the size ofa whole administrative region of the city's integration. Covering over $97 \%$ of the communities in the city, it officially came into service in 2016 , providing push-totalk terminal for the aged who is over 80 years old and who is in need of that. In face of the increasing demand of old-age care, Zhoushan's 2.0 version of intelligent old-age care system - the intelligent old-age care center of the new districtin the Zhoushan isles - has been put into use. Based on the original platform for the intelligent old-age care of Zhoushan, it is a materialized platform created by the joint efforts of Zhoushan Civil Affairs Bureau and the branch office of China Telecom in Zhoushan, making the aged able to experience a kind of old-age care that is informationlized

\section{DiLEMMA}

Zhoushan presents a positive prospect on developing international intelligent old-age care, but owing to both some historical and existing reasons, it's still faced with lots of difficult positions in building the sea garden where people could enjoy international intelligent old-age care. First of all, its level of internationalizationis far lower than those first- and second-tier cities in China, with its infrastructures like transportation and information not well-developed. Furthermore, the development and creation of the network for the intelligent old-age care are still in the early stages, and the elderly don't have much experience on the usage of the products for old-age care.

\subsection{The Level of Internationalization is Low}

As an important city of the Yangtze River Delta region, Zhoushan is developing fast thanks to the more and more attention government has paid to the island areas and marine economy in recent years. But in terms of its overall level of development, Zhoushan's economic aggregate is not large enough and the level of internationalization is inferior to that of other cities in the Yangtze River Delta region. Take the airport, which is a symbol of internationalization, as an example, the airport of Zhoushanis smaller and has fewer flights, shorter flight courses, making it inadequate for an international airport. For those foreign friends who come to visit Zhoushan, the transports to get there are so limited that they have to transfer. In addition, the international public services of government are insufficient, such as the shortage of English signs and English information for direction in lots of public services sectors. This phenomenon also appears in hospitals where some medicals services are involved, banks 
where people handle their personal businesses, and in other places like buses, scenic areas, stores where special services are provided respectively. The procedures that are involved in those occasions are familiar to the local people but unfamiliar to foreigners. Besides, the popularizing rate of English for common citizens is not high and consequently the relevant guidance to help the foreigners is available enough.

\subsection{The Infrastructures like Transportation are not Well-Developed}

Restricted by the natural environment and due to some history factors, the construction of various infrastructures in Zhoushan island start relatively late. At present, what Zhoushan has is only a crosssea bridge which connects it with Ningbo and neither train nor light rail is available there. Remarkable improvements have been achieved on the condition of the local infrastructures for information and transportation owing to the support of pertinent policies and the joint efforts of government and people of Zhoushan. Nevertheless, generally speaking, the penetration of the roads in the island is not sufficient and the density of road network is not high enough, indicating there is still a gap for Zhoushan to fill to be an intelligent city for old-age care.

\subsection{The Technological Development is Still in the Early Stages}

The cores of intelligent old-age care are Big Data, the Internet of Things and Cloud Center, but in our country, there are only several technical development corporations which concentrated on developing the network for intelligent old-age care. The Action plan for the development of the intelligent old-age care system (2017-2020) issued by the State Council points out that by 2020, we are going to basically form an industrial system for intelligent old-age care that will cover a full lifecycle, establish over 100 demonstration bases of the application of intelligent old-age care and nurture over 100 leading enterprises that will play an exemplary role in the industry. According to the incomplete statistics from the Prospective Industry Research Institute, within the total of 31 corporations which is involved in intelligent old-age care industry, there are 12 of listed company focusing on the founding of the service platform for intelligent old-age care. The statistics discovers that most of the firms offer the integrated solution plan and join in the intelligent old-age care industry by cooperating with government departments (like the Civil Affairs Bureau). But their technical developments are still in the trial phase, breaking in with the needs of citizens. The development of the technology of the network for the intelligent old-age care and the very founding of it are very complicated and are huge projects. Situated at an island with a more complicated geographical conditions and limited level of imformatization, Zhoushan is challenged in developing the technology of its intelligent old-age care.

\subsection{There is a Common Gap among the Elderly on Accessing the Information}

Human's health condition would get worse with age, and our cognitive competence like the ability to learn a new skill and memorize things will be affected as well. Those elderly people, as the generation of the middle and upper period in the last century, accepted less digital information when they were young. This is a worldwide phenomenon, which also happened in America, where currently owns the most advanced Internet technology across the world. The America popularized the usage of Internet in the 90's, when relatively less opportunities to access the digital information are offered for the generation then, and whose educational level was not high as well, limiting their ability to accept new knowledge and prolonging their adaptation time. One difficult position for the intelligent old-age care system is that the elderly have problems using the products providing the intelligent old-age care.

\section{RECOMMENDED METHODS}

Not only does Zhoushan have the potential to become international, but also can it highlight its strength against the background of world population aging by seizing its opportunity in the tide of silver economy. Through deepening its reforms and trials in free trade zone, it could contribute to the construction of Zhoushan city, prompting the founding of the center for intelligent old-age care in China, which is going to be elevated into the center in the world in the future

\subsection{Improve the Level of Internationalization of Zhoushan}

Zhoushan, as far as it goes, is not even close to an international metropolis, nor its condition of 
hardware or software facilities, but it still should combine its own strengths and do well in the matter of the cultural exchange and construction of industry, which may do some help. All the international conferences that are presently set to be held in Zhoushan like the International Petroleum and Natural Gas Enterprises Conference, the International Islands Tourism Conference and the Tour of Zhoushan Island could be the helpful instruments for Zhoushan to publicize its special features. By letting the government and media play their role more competently and strive for making Zhoushan the host of more international conferences, more exchange opportunities will be rendered available. Apart from that, the government should uplift the consciousness of international public service and upgrade the level of that by pasting English signs and informational directions in the public service sectors, which includes occasions like hospitals, where some medicals services are involved, banks where people handle their personal businesses, and in other places like buses, scenic areas, stores where special services are provided respectively. Those practices are the facilitators for Zhoushan to go globally.

\subsection{Enhancing the Construction of Transportation and Information Network}

In terms of transportation construction, it is clear the development of the international old-age care center can't proceed without the support of the airport and other transportation facilities, justifying the reason of accelerating the construction of public transportation. In terms of information network construction, the first task is to arrange the very construction properly, including FTTH and the construction of the information service platform that has content. The second task is to ensure the resource guarantee is in position, which means that the government's function of macroscopic readjustment and control is given full play, coordinating the economic and social development in urban and rural areas. Meanwhile, government should paly a leading part in investment and development, arranging certain percentage of fiscal fund for assisting agriculture and for agricultural development and to advance the construction of agriculture information.

To build information infrastructures in rural area, the government is the way of reliability on the hardware side, in which some practical actions need to be adopted, some projects like "Information to the Rural Areas" and "Broadband to the Rural Areas" needed to be carried out. These practices will help subsidize the famers in a moderate way when they are buying phones, computers, lowering the threshold of using them and ensuring their being able to buy computers and access the Internet without bearing the changes in the grid's price. As regards the software for the intelligent old-age care carried out in rural areas, especially the building of the talent team, there are several aspects we can start with. To nurture the talent for the old-age care center, which is mentioned earlier this essay, the civil-service job with personnel establishment should be set up to attract a mass of youngsters who are conversant with computer to take this office, while for the medical service center, which may take a longer cycle to train new staff, the means of subjecting the current medical workers to the training of computer networking technology could be adopted.

\subsection{Expediting the Technology Research and Development, Driving the Intelligent Upgrade}

The research and development of the technology is the pivotal power of the whole intelligent old-age care industry. In order to be an international center for intelligent old-age care, it is essential to strengthen the relevant technological development and research of the application for the intelligent old-age care, industrialize the very products of it and continuously improve the quality and the brand. To accomplish that, the government departments should first introduce relevant talents, providing policy guarantees by bringing about innovations in systems and mechanisms. In the next place, based on the incentive policies and against the background of mass innovation, relevant technological corporations should begin to focus on lifting their scientific and technological level, unceasingly improve their capability of independent innovation, voluntarily assume their social responsibility and create a more beautiful world.

\subsection{Narrowing the Information Gap Existing Among the Aged}

Throughout the world, the information gap for the moment, commonly exist among the aged. For the solution to this sort of problem, the government departments should first establish bases where information could be popularized among the aged, nurture relevant staff perform this popularizing task, set up consultative panels for the convenient explanations for the questions of the aged. Secondly, the terminal devices should be easily operated and be equipped with the function by which one button could set everything ready. By pushing forward the projects of combining the colleges with local education governments, the young volunteers could be enrolled in the team of science popularization, which, on the one hand, provides the service of information popularization for the 
aged who will be experiencing the intelligent old-age care in Zhoushan, on the other hand, gives the young people some ideas of the innovativeness, practicability of intelligent old-age care system. Through the interpretation and publicity by the young people, Zhoushan's center of intelligent old-age care will go out of China into the world.

Conclusion: With the arrival of the world population aging, the Big Data becomes an important technological means for the aging society. "The Aging plus the Big Data" is of great significance for the solution to the aging problems and for the sound development of the aging society. By right of the advantageous geographic position and the strong support of policies, Zhoushan has the potential and capability to become an international garden city and to develop into an international center for intelligent old-age care, discovering new opportunities in the tide of silver economy.

\section{REFERENCES}

[1] D. Maeda, K. Teshima, H. Sugisawa, Y. S. Asakura. Ageing and health in Japan[J]. Journal of CrossCultural Gerontology, 1989, 4(2).

[2] Ebrahim S. Public health implications of ageing.[J]. Journal of Epidemiology \&amp; Community Health, 1998, 51(5).

[3] Yoosik Youm, Edward O Laumann, Kenneth F Ferraro, Linda J Waite, Hyeon Chang Kim, Yeong-Ran Park, Sang Hui Chu, Won-takJoo, Jin A Lee. Social network properties and self-rated health in later life: comparisons from the Korean social life, health, and aging project and the national social life, health and aging project [J]. , 2014, 14(1).

[4] Mussel white Charles, Scott Theresa. Developing A Model of Mobility Capital for An Ageing Population. [J]. International journal of environmental research and public health, 2019, 16(18).

[5] Vogel sang Eric M, Raymo James M, Liang Jersey, Kobayashi Erika, Fukaya Taro. Population Aging and Health Trajectories at Older Ages.[J]. The journals of gerontology. Series B, Psychological sciences and social sciences, 2019, 74(7).

[6] "智慧养老": 养老模式的新兴路径 [J].经济展望, 2013:197.

[7] 刘公博.智慧城市背景下智慧社区养老模式研究[J].中国集体经济, 2019,(29):3-4.

\section{AUTHORS' BIOGRAPHY}

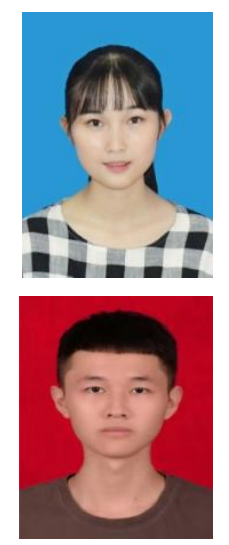

Yanhong Wu, was born in 1998, China, now studies in Zhejiang Ocean University.

Yahao Xu, was born in 1999, China, now studies in Zhejiang Ocean University.

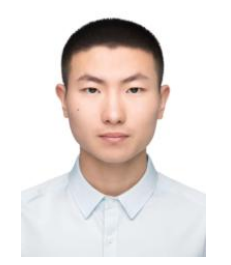

Jingxing Wang, was born in 1999, China, now studies in Zhenjiang Ocean University.

Citation: Yanhong Wu, et.al. "Explorationson Seizing Opportunities in the Tide of Silver Economy via Intelligent Old-Age Care System from International Perspective" International Journal of Humanities Social Sciences and Education (IJHSSE), vol 7, no. 2, 2020, pp. 20-25. doi: http://dx.doi.org/10.20431/2349-0381. 0702004.

Copyright: (C) 2020 Authors. This is an open-access article distributed under the terms of the Creative Commons Attribution License, which permits unrestricted use, distribution, and reproduction in any medium, provided the original author and source are credited. 